\title{
Junction Configuration-Induced Mechanisms Govern Elastic and Inelastic \\ Deformations in Hybrid Carbon Nanomaterials
}

\author{
Rouzbeh Shahsavari $^{1,2,3,{ }^{*}}$ and Navid Sakhavand ${ }^{1}$ \\ ${ }^{1}$ Department of Civil and Environmental Engineering, Rice University, Houston, TX 77005 \\ ${ }^{2}$ Department of Material Science and NanoEngineering, Rice University, Houston, TX 77005 \\ ${ }^{3}$ Smalley Institute for Nanoscale Science and Technology \\ *Corresponding author email: rouzbeh@rice.edu
}

\begin{abstract}
Although various hybrid 3D carbon-based architectures are reported by covalently connecting low dimensional carbon allotropes, the effect of junction configuration on deformation and mechanical properties of the hybrid carbon architectures remain elusive. Here, we focus on Pillared Graphene Nanostructure (PGN) as a model system with symmetric and asymmetric junctions to explore its diverse elastic and inelastic properties via first-principles and molecular dynamics simulations. By introducing heptagonal and octagonal rings in the junctions, our findings demonstrate that in contrast to the stacked of graphene sheets, which exhibit weak out-of-plane properties, both junction types impart a cooperative two-regime deformation mechanism that provides a number of superior characteristics in PGN, including $3 \mathrm{D}$ balance of strength and toughness as well as an outstanding $\sim 42 \%$ out-of-plane ductility preceding the failure. Furthermore, asymmetric junctions impose wrinkles in the PGN sheets, which add extra in-plane flexibility and shear compliance, result in a nearly zero/negative in-plane Poisson's ratio in PGN, and cause the octagonal rings to act as hotspot for initiating of fracture. Our results provide the first atomistic "lens" on fundamental understanding of junction-induced deformation mechanisms in pillared graphene and can potentially provide a new phase space to better control and design mechano-mutable hybrid carbon nanostructures.
\end{abstract}


Keywords: Pillared graphene, 3D nanostructures, mechanical properties, synergistic effect.

\section{Introduction}

Low dimensional carbon-based materials such as 1-dimensional (1D) carbon nanotubes (CNTs) and 2D graphene exhibit several attractive physics and fundamental properties [1]. However, there is a high anisotropy with such $1 \mathrm{D}$ and 2D nanomaterials. For instance, while graphene has high in-plane stiffness $(>1 \mathrm{TPa})$ and thermal conductance, owing to the strong carbon-carbon bonds [2], its out-of-plane direction stiffness and thermal conductance are considerably low, due to the weak Van der Waals forces [3]. Similarly, the high stiffness and thermal conductance of CNTs are limited to axial directions [4]. This restriction has stimulated researcher's to search and create hybrid nanomaterials that can leverage the best aspects of their constituents' and overcome the intrinsic limitations of their parent structures. Carbon nanopeapods [5-7], carbon nanobuds [8], periodic graphene nanobuds [9], nanotube-drive carbon foams [10], and pillared graphene [11] are examples of recently introduced hybrid nanostructures. Among these, Pillared Graphene Nanostructure (PGN) is a 3D structure that is created by fusing graphene sheets with carbon nanotubes via covalent bonds. Anisotropic aspects of graphene and carbon nanotubes, as 2D and 1D structures, are leveraged to confer an interesting 3D network with multifunctional properties such as high mechanical [12] and thermal [13] properties that are comparable to its constituents. There are several works in the literature that each has focused on certain feature(s) of PGN. For instance, Wang et al. [14] modeled nanoindentation tests along the out-of-plane direction of PGN. While their results provide insights on compressive behavior of PGN, the key benefits of PGN (to our knowledge) is in tensile stress since PGN with long tubes and flats sheets are subject to quick buckling in compression. Niu et al. [15] investigated the influence of defects such as (5577) point defects and grain boundaries on mechanical properties of PGN 
along the out-of-plane direction. This work only considered a single junction made of one CNT and graphene, which does not allow realizing more delicate and non-intuitive features such as junction-junction correlations, which are only manifest when studying larger supercells of PGN. Similarly, Sasaki et al. [16] studied the shear behavior of PGN junctions. In brief, while previous works have each their own merits, to our knowledge there has been no rigorous and complete study on the elastic and inelastic properties of PGN including full elastic tensors, bond strains, strength, ductility and toughness in all three-dimensional directions.

Other multifunctional properties of PGN such as gas storage [11], gas separation [17], and electronic transport [18] are also predicted. These unique 3D properties of PGN have encouraged many attempts to synthesize PGN [19] and even fabricate nano devices such as PGN-based super capacitors [20]. From the design standpoint of PGN, inter-pillar distance (PD) and pillar length (PL) are key parameters defining various properties of PGN. It has been shown that changing these parameters yield different properties, thus enabling purpose-specific synthesis of PGN [12, 13, 21]. The effect of nanotube size is also studied [22].

Another key characteristic of PGN is the junction. Despite the critical role of the junction in covalently connecting the $1 \mathrm{D}$ and $2 \mathrm{D}$ constituents in PGN, they have received relatively less attention compared to PD and PL. The nanotubes are disconnected at the junctions, hence providing structural flexibility, phonon scattering, and Kapitsa effect for PGN [23]. In this context, while there are numerous ways to create the junctions [24], to our knowledge all the explored properties of PGN are based on the assumption of symmetric junctions, i.e. six heptagonal rings around the junction of $\mathrm{CNT}$ and graphene, in addition to the conventional hexagonal rings. In this paper, we introduce a new prototype of PGN via first-principles 
calculations containing three octagonal rings, leading to asymmetric junctions. Next, we compare the mechanical properties of two PGN prototypes, which are composed of symmetric and asymmetric junctions using molecular dynamics (MD) simulations. Our study focuses on evaluating stiffness, stress-strain plots, bond strains and inelastic deformation mechanisms for each of the PGN prototypes along the $\mathrm{X}, \mathrm{Y}$, and $\mathrm{Z}$ directions.

\section{Computational Methods}

\subsection{General Atomistic Simulation Approaches}

Density functional theory (DFT) [25] was used for fusing the CNT and graphene based on Becke's three-parameter hybrid functional combined with Lee-Yang-Parr correlation functional (B3LYP) with $6-31+\mathrm{G}^{*}$ basis set. These calculations were conducted using the Gaussian 09 suite of programs [5]. The convergence criterion is the Gaussian default convergence criterion, which is 0.00045 Hartree/Angstrom and 0.0003 Hartree/Angstrom for the maximum and root mean square (RMS) of forces, respectively, and 0.0018 and 0.0012 Anstrom for the maximum and RMS displacements, respectively. These default criteria are very stringent and examine the mean and RMS variance of both forces and displacements. LAMMPS code [26] was used for MD simulations. The well-known Adaptive Intermolecular Reactive Empirical Bond Order (AIREBO) [27] potential was used for carbon-carbon bonds. Following [28] [29], we used a switch function parameter $r_{C C}=1: 92 \AA$. This choice of $r_{C C}$ in the AIREBO potential is validated by comparing the density functional theory calculations and MD simulations for the stress-strain curves in pristine graphene in either the armchair or zigzag direction [29]. Before calculating mechanical and thermal properties, each PGN prototype was relaxed for $1 \mathrm{~ns}$ under isothermal-isobaric ensemble (NPT) at zero pressure and temperature with Nose-Hoover 
thermostat $[30,31]$ for the time integration. All MD simulations were conducted with 1 femtosecond time step. The Visual Molecular Dynamics (VMD) virtualization package [32] was used for visualizations. Stress contour plots were created by Atomeye, an atomistic configuration viewer package [33].

\subsection{Calculation of Mechanical Properties.}

Prior to calculating the elastic properties, we perform energy minimizations by conjugate gradient method as implemented in LAMMPS to fully relax the PGN prototypes. To calculate the full elastic constants tensors, we adopted the stress-strain approach [9] to apply six strains to the cell coordinates. For each strain, the system was relaxed while the box dimensions were fixed. Then, by calculating the stress tensor via virial theorem [34] a linear system was constructed relating stresses to strains by generalized Hooke's law in linear elasticity

$$
\left[\begin{array}{l}
\sigma_{1} \\
\sigma_{2} \\
\sigma_{3} \\
\sigma_{4} \\
\sigma_{5} \\
\sigma_{6}
\end{array}\right]=\left[\begin{array}{llllll}
C_{11} & C_{12} & C_{13} & C_{14} & C_{15} & C_{16} \\
C_{12} & C_{22} & C_{23} & C_{24} & C_{25} & C_{26} \\
C_{13} & C_{23} & C_{33} & C_{34} & C_{35} & C_{36} \\
& & & C_{44} & C_{45} & C_{46} \\
& & & & C_{55} & C_{56} \\
\text { Sym } & & & & & C_{66}
\end{array}\right]\left[\begin{array}{l}
\epsilon_{1} \\
\epsilon_{2} \\
\epsilon_{3} \\
\epsilon_{4} \\
\epsilon_{5} \\
\epsilon_{6}
\end{array}\right]
$$

In above, $\sigma$ and $\varepsilon$ denote the stress and strain while $C_{i j}$ represent the elastic constant components shown in contracted Voigt notation. By applying a nonzero strain in Eq. (1) and calculating stresses, one can obtain a column of elastic constants. Repeating this procedure for all the six strains covers the whole elastic tensor. In this approach, since off-diagonal components appear twice in the calculations, we take the average of the two equivalent off-diagonal terms to have a better estimate. Finally we use an orthogonal matrix factorization and the best least square fit to find the elastic constants via minimization of [35] 


$$
\left|\left(\sigma_{\mathrm{i}}-\sigma_{\mathrm{r}}\right)-C_{\mathrm{ij}}\left(\varepsilon_{\mathrm{i}}-\varepsilon_{\mathrm{r}}\right)\right|
$$

where $\sigma_{\mathrm{r}}$ and $\varepsilon_{\mathrm{r}}$ are residual stress and residual strain, $\left(\varepsilon_{\mathrm{i}}-\varepsilon_{\mathrm{r}}\right)$ is the applied strain, and the repeated indices denote Einstein summation rule. In this method, the uncertainty in $C_{i j}$ values will be minimized with improved overall accuracy. This method has been highly successful to predict elastic constants of several crystalline materials [35]. We applied 5\% strain in all six directions to obtain the elastic constants.

Once an elastic tensor, $C$, is calculated, we got the compliance tensor via $S=C^{-1}$. Then, the Young moduli along different orthogonal directions reads [36]

$$
E_{1}=\frac{1}{s_{11}}, E_{2}=\frac{1}{S_{22}}, E_{3}=\frac{1}{s_{33}}
$$

In equation (3), $S_{11}$ refers to the first component of the compliance tensor and so on, and the indices 11, 22 and 33 represent $\mathrm{X}, \mathrm{Y}$ and $\mathrm{Z}$ directions, respectively. Once a full compliance tensor is obtained, the 3D elastic Young moduli along any arbitrary direction in space are computed via rotation of a compliance matrix [37].The anisotropic Poisson's ratios are also obtained from the compliance tensor via

$$
v_{i j}=-\frac{s_{i j}}{s_{i i}} \quad i, j=1,2,3 \quad \text { and } \quad i \neq j
$$

To obtain the orthogonal shear moduli of PGN and in view of their small off-diagonal terms (see Section 3.3), we approximate the elastic constants tensor of PGN with that of an orthotropic material [36]

$$
\left[\begin{array}{cccccc}
C_{11} & C_{12} & C_{13} & 0 & 0 & 0 \\
C_{12} & C_{22} & C_{23} & 0 & 0 & 0 \\
C_{13} & C_{23} & C_{33} & 0 & 0 & 0 \\
& & & C_{44} & 0 & 0 \\
& & & & C_{55} & 0 \\
\text { Sym } & & & & & C_{66}
\end{array}\right]
$$


Then, orthogonal shear moduli can be obtained from

$$
G_{12}=\frac{1}{S_{66}}, G_{13}=\frac{1}{S_{55}}, G_{23}=\frac{1}{S_{44}}
$$

here, the $G_{12}, G_{13}$ and $G_{23}$ refer to $G_{X Y}, G_{X Z}$ and $G_{Y Z}$, respectively.

Beyond elasticity, the inelastic properties (stress-strain plots) in each direction were calculated by applying strains at the increments of $1 \%$ in the corresponding direction while restraining the system against displacement in the other directions. The system was then allowed to relax and the stress tensor was calculated via the virial theorem [34]. Note that stress-strain calculations were also repeated with $0.1 \%$ strain increments, but did not show a noticeable difference with the results from $1 \%$ strain increments. All the elastic and inelastic properties were obtained by energy minimization at $0 \mathrm{~K}$ to exclude thermal effects.

\section{Results and Discussion}

3.1 Creation of the Symmetric and Asymmetric Junctions. Figure 1 shows a general schematic picture of 3D PGN made of 1D CNNT and 2D monolayer graphene sheets. A key attribute of the PGN prototypes is the junction between the tubes and sheets for which there are several possibilities depending on the tube radius and chirality [24]. In our study, the symmetric and asymmetric junctions were created computationally by bringing a $(6,6)$ single wall CNT close to defected graphene monolayer sheets (Figure 1e,f) to form a 3D building block. The specific forms of the defects in graphene sheets, shown in Figure 1a,b, are consistent with the $(6,6)$ CNT to satisfy the $\mathrm{sp}^{2}$ configuration for all the atoms at the junction. We fused the nanotubes and the sheets in Figure 1c,d by fully optimizing the system with DFT calculations to create the basic 3D building blocks (Figure 1e,f), which include symmetric and asymmetric junctions. While the former (symmetric junction) includes 6 heptagons aligned symmetrically 
with a $60^{\circ}$ apart (Figure 1e,g), the latter contains 3 octagons, which form an asymmetrical (along the X-axis) triangular-shape junction, due to the odd number of the octagons (Figure 1f,h).

Note that in both symmetric and asymmetric junctions, the final configurations are consistent with the Euler's polygon theorem [24, 38], i.e. the number of faces $F$, vertices, $V$, Edges $E$ and Genus, $G$, are related as $F+V=E+2-2 G$ or $6(E-F-V)=12(G-1)[6]$; CNT-graphene junction is a closed surface of genus $2(G=2)$. Thus, CNT and graphene must share a total bond surplus of 12 (6 each) [11]. PGN-Sym satisfies this criterion by 6 heptagons, each having a +1 bond surplus; PGN-Asym by 3 octagons, each having a surplus of +2 .

(a)

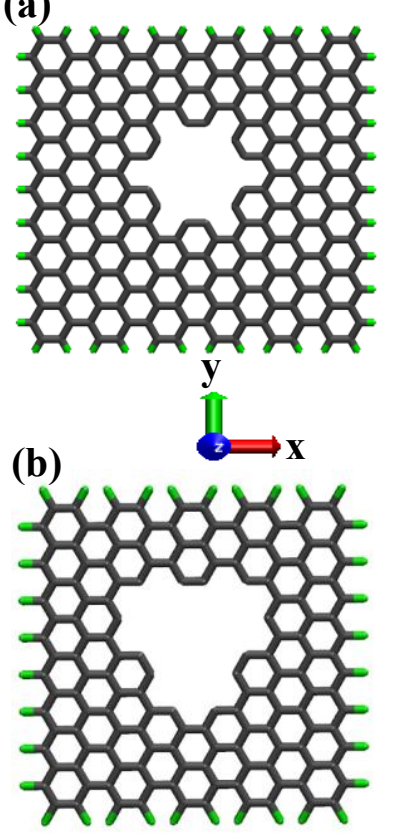

(c)

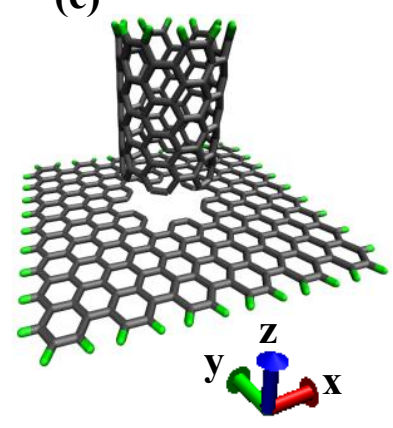

(d)

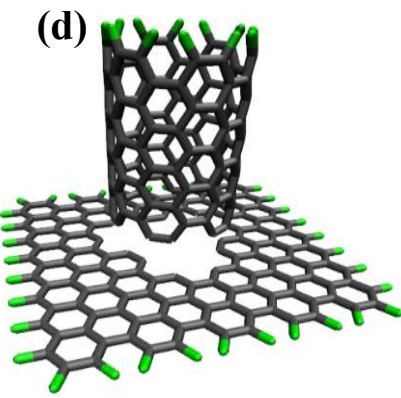

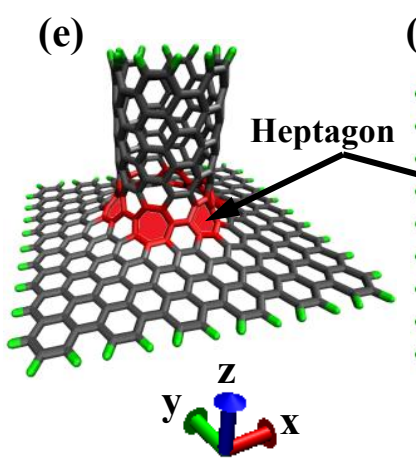

(f)

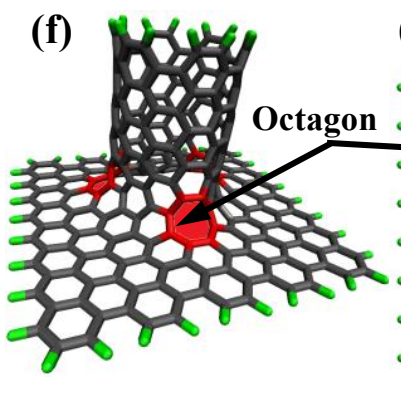

(g)

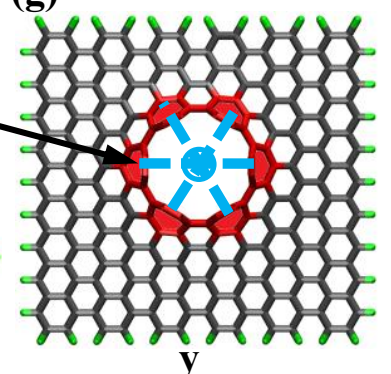
$\mathbf{y}$

(h)

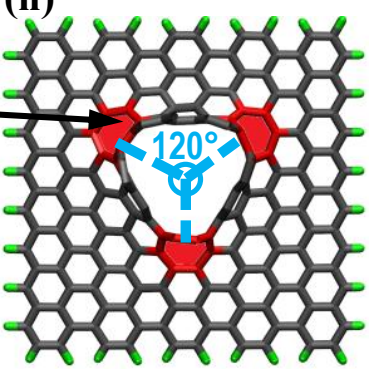

Figure 1. (a-b) Two different defects are made in a graphene sheet. The dangling bonds are protonated to satisfy the $\mathrm{sp}^{2}$ configuration. (c-d) A $(6,6) \mathrm{CNT}$ is vertically aligned close to the defected graphene. (e-f) The final building blocks are obtained by DFT optimization. Nonhexagonal rings are shown in red. The junctions satisfy Euler's polygon theorem. (g) A top view of the symmetric junction with 6 heptagons, which are aligned $60^{\circ}$ apart. (h) A top view of the 
asymmetric junction with 3 Octagons, which are $120^{\circ}$ apart. This junction is symmetric only along the $\mathrm{Y}$-axis.

3.2 PGN Unit Cells with Periodic Boundary Conditions. To create the 3D unit cells of PGN with periodic boundary conditions, the 3D building blocks in Figure 1e,f were extended to 3D pillared motifs using a series of transformations and rotations. The final atomic structures and cell dimensions were obtained via relaxing the unit cells via MD simulations under an isothermal-isobaric ensemble. The first PGN, we term it PGN-Sym (Figure 2a), was constructed using the building block of Figure 1e with 6 heptagons per junction. The second PGN, we term it PGN-Asym (Figure 2b), was constructed using the building block of Figure 1f. In both PGNs single-wall carbon nanotubes support the graphene sheets forming a 3D nanostructure. Due to the in-plane symmetry of the junctions of PGN-Sym, the sheets connecting the nanotubes are flat (Figure 2a). However, in the case of PGN-Asym, the octagon rings of the junctions are located at the vertices of a somewhat triangular-type defect (Figure 1h). Thus, each junction is locally symmetric along the $\mathrm{Y}$-axis (zigzag) but asymmetric along the $\mathrm{X}$-axis (armchair). Such specific junction alignment causes the graphene sheets to be wrinkled in both $\mathrm{X}$ and $\mathrm{Y}$ directions after MD relaxation of the unit cell (Figure $2 b$ ). Therefore, even in the absence of external force, both the junctions and the graphene sheets are slightly stressed due to the imposed curvature. Such wrinkles have not been observed in previous 3D carbon structures [11]. 


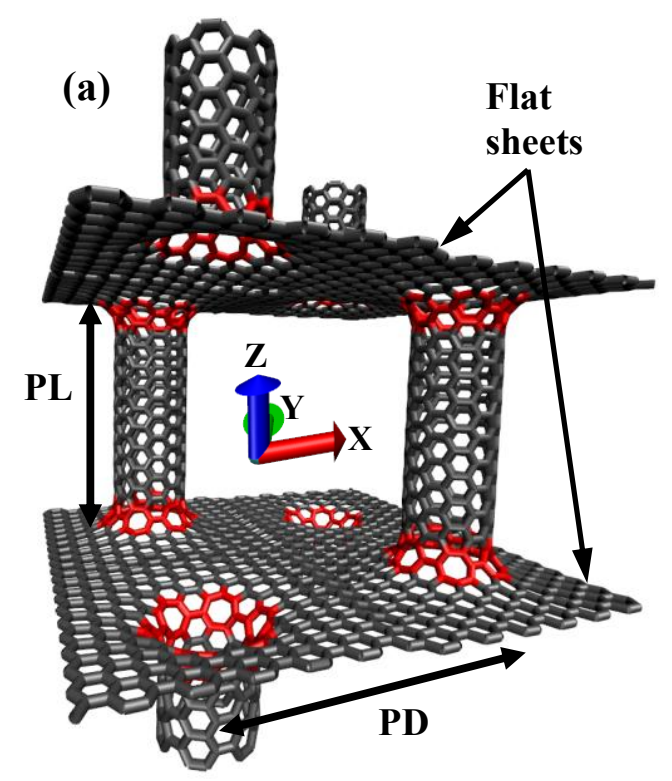

PGS-Sym

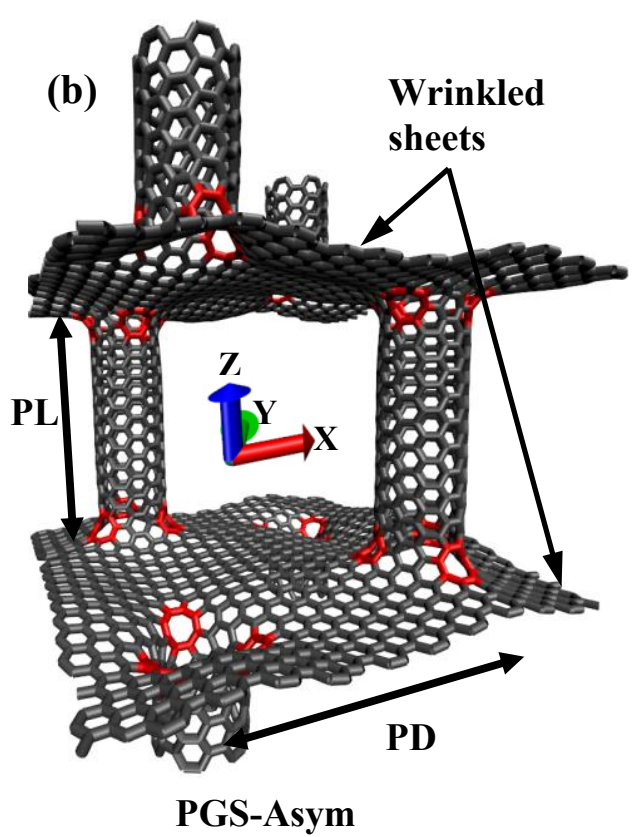

PGS-Asym

Figure 2. (a) a unit cell of PGN-Sym prototype. The junctions include 6 heptagons shown in red. Symmetry of the junctions in $\mathrm{X}$ - and $\mathrm{Y}$-axes causes the sheets to be flat. (b) a unit cell of PGNAsym. The junctions exhibit symmetry along the $\mathrm{Y}$-axis but asymmetry along the $\mathrm{X}$-axis, thereby leading to wrinkled sheets in PGN-Asym. Each unit-cell in (a-b) contains four columns and 2 sheets.

Figure 2 clearly depicts the pillar distances (PD) and pillar lengths (PL), which both affect mechanical [12] properties of PGN. To focus our study on the effect of the junctions, we built PGN-Sym and PGN-Asym with almost similar geometrical dimensions. Detailed structural information of both PGNs are shown in Table 1. Because of the wrinkled sheets, PGN-Asym with similar PD and PL to PGN-Sym includes more number of atoms than PGN-Sym. While both PGNs have similar pillar length, the wrinkled sheets of PGN-Asym make the unit-cell to be slightly longer along the tubes (Z-axis) than PGN-Sym. In what follows, we explore how the 
symmetric and asymmetric junctions affect the mechanical properties of these two PGN prototypes.

Table 1 - Number of atoms, structural details of four PGN prototypes.

\begin{tabular}{lllllllll}
\hline $\begin{array}{l}\text { PGN } \\
\text { Prototype }\end{array}$ & $\begin{array}{l}\text { number } \\
\text { of atoms }\end{array}$ & pillar length, & PL $(\mathrm{nm})$ & $\mathrm{PD}(\mathrm{nm})$ & $\mathrm{Lx}$ & $\mathrm{Ly}$ & $\mathrm{Lz}$ & \multicolumn{2}{l}{ sistance, } & \multicolumn{2}{l}{ unitcell size $(\mathrm{nm})$} & \multicolumn{2}{c}{ surface $\left(\mathrm{m}^{2} / \mathrm{g}\right)$} & $\begin{array}{c}\text { density } \\
\left(\mathrm{kg} / \mathrm{m}^{3}\right)\end{array}$ \\
\hline PGN-Sym & 2736 & 2.45 & 2.64 & 5.0 & 4.8 & 5.9 & 2257.7 & 398.1 \\
PGN-Asym & 2784 & 2.43 & 2.64 & 5.0 & 4.8 & 6.2 & 2251.7 & 385.5
\end{tabular}

\subsection{Elastic properties}

We calculated the elastic properties by introducing the effective (gross) areas and then by introducing an equivalent area in which the voids in the nanostructure are subtracted from the effective area. In other words, the equivalent area is the net atomic area. While the use of atomic areas usually enables determining the limiting mechanisms and are more appropriate for comparison to parent structures (CNT and graphene), the overall gross (effective) cross sectional area is important from material design perspectives and continuum analysis of the stresses. Both the effective and equivalent elastic moduli are listed in Figure 3a, and are shown schematically in Figure 3c. First, we discuss the effective moduli. Equations (7-8) show the calculated elastic constant tensors (stiffness matrices) of PGN-Sym and PGN-Asym in GPa. While the elastic tensors are suggestive of an anisotropic behavior, the extent of this anisotropy differs from PGNSym to PGN-Asym. To better understand this behavior, the variation of Young's moduli are calculated along any arbitrarily direction in space via rotation of elastic stiffness tensors. Figure $3 \mathrm{~b}$ shows the data in the form of $3 \mathrm{D}$ contours of elastic moduli, which upon linking with the PGN nanostructures provide important physical insights to be discussed next. 
First, given the minimal variation of the elastic moduli in the planar directions, both PGNs demonstrate in-plane mechanical symmetry. The out-of-plane moduli are much lower than the in-plane ones for both PGN. This originates from the flexibility of the sheets in the out-ofplane directions (Z-axis). It has been previously shown that reducing PD (similarly PL), decreases contribution of the sheets (tubes), thus increasing the out-of-plane (in-plane) elastic moduli [12]. Our analysis shows that for constant PD and PL, the out-of-plane moduli are similar in PGN-Sym and PGN-Asym. However, regarding in-plane direction, while both PGNs show inplane symmetry, PGN-Asym exhibits lower in-plane stiffness and uniformity compared to PGNSym. More precisely, the in-plane moduli of PGN-Asym are $\sim 18 \%$ lower than that of PGN-Sym. This is because the wrinkled sheets of PGN-Asym provide flexibility for the in-plane directions, thus reducing the in-plane moduli (c.f. Movies S1 and S2). This reveals that in addition to PD and PL, the type of the junction can have a significant impact on the elastic properties of the PGN, which is oftentimes overlooked in the literature.

We also studied the effect of (a)symmetric junctions on orthogonal shear moduli. As shown in Table 2, it turns out that while asymmetry results in $\sim 7 \%$ and $\sim 19 \%$ reduction in the inplane $\left(G_{\mathrm{XY}}\right)$ and out-of-plane shear moduli in X-Z plane $\left(G_{\mathrm{XZ}}\right)$, respectively, it leads to $\sim 41 \%$ increase in the out-of-plane shear modulus in Y-Z plane $\left(G_{\mathrm{YZ}}\right)$. This trend is counterintuitive given the both reduction and increase in two out-of-plane $\mathrm{X}-\mathrm{Z}$ and $\mathrm{Y}-\mathrm{Z}$ shear moduli. The reason lies in the fact that the shear deformation in the $\mathrm{Y}-\mathrm{Z}$ plane activates more stretch in the $\mathrm{C}-\mathrm{C}$ bonds of the octagons, compared to the X-Z planes (see Figure 1e,f). The behavior can be also understood from the stress concentrations imposed by octagons, to be discussed shortly. Nevertheless, for both PGN prototypes, the in-plane shear modulus, $G_{\mathrm{XY}}$, is higher than out-of- 
plane shear moduli ( $G_{\mathrm{XZ}}$ and $\left.G_{\mathrm{YZ}}\right)$ due to the small lateral stiffness of the tubes and flexibility of the junctions.

\begin{tabular}{|c|c|c|c|c|c|c|}
\hline \multirow{5}{*}{$\mathrm{C}_{\mathrm{PGN}-\mathrm{Sym}}=$} & \multirow[t]{5}{*}{86.85} & \multirow{3}{*}{$\begin{array}{l}16.84 \\
81.62\end{array}$} & \multirow{2}{*}{$\begin{array}{l}10.12 \\
9.83\end{array}$} & \multirow{2}{*}{$\begin{array}{l}-1.12 \\
-0.87\end{array}$} & \multirow{2}{*}{$\begin{array}{l}-0.85 \\
-1.10\end{array}$} & \multirow{2}{*}{$\begin{array}{l}-1.77 \\
0.77\end{array}$} \\
\hline & & & & & & \\
\hline & & & 12.21 & -0.29 & -0.24 & -1.47 \\
\hline & & & & 2.34 & 0.00 & 0.02 \\
\hline & & \multicolumn{2}{|l|}{ Sym } & & 3.10 & $\begin{array}{l}0.00 \\
30.12\end{array}$ \\
\hline \multirow{5}{*}{$\mathrm{C}_{\text {PGN-Asym }}=$} & \multirow[t]{5}{*}{69.11} & 6.05 & 8.32 & -1.75 & -1.50 & -0.72 \\
\hline & & 68.82 & 8.97 & -1.13 & -1.31 & 0.02 \\
\hline & & & 11.88 & -0.18 & -0.22 & -1.08 \\
\hline & & & & 3.34 & -0.01 & -0.13 \\
\hline & & \multicolumn{3}{|l|}{ Sym } & 2.90 & $\begin{array}{c}-0.12 \\
24.40\end{array}$ \\
\hline
\end{tabular}

Table 2 - Effective and equivalent areas for PGN prototypes.

\begin{tabular}{|c|c|c|c|c|c|c|}
\hline & $E_{\mathrm{X}}$ & $E_{\mathrm{Y}}$ & $E_{\mathrm{Z}}$ & $G_{\mathrm{XY}}$ & $G_{\mathrm{XZ}}$ & $G_{\mathrm{YZ}}$ \\
\hline \multicolumn{7}{|c|}{ effective moduli (GPa) } \\
\hline PGN-Sym & 77.03 & 72.25 & 10.17 & 29.88 & 3.08 & 2.32 \\
\hline PGN-Asym & 61.84 & 61.25 & 9.83 & 24.28 & 2.85 & 3.28 \\
\hline \multicolumn{7}{|c|}{ effective moduli (GPa) } \\
\hline PGN-Sym & 670.95 & 629.30 & 137.81 & 260.25 & 41.75 & 31.45 \\
\hline PGN-Asym & 562.43 & 557.04 & 130.97 & 220.83 & 37.94 & 43.71 \\
\hline \multicolumn{7}{|c|}{ effective area $\left(\mathrm{nm}^{2}\right)$} \\
\hline & $L_{\mathrm{Y}} \cdot L_{\mathrm{Z}}$ & $L_{\mathrm{X}} \cdot L_{\mathrm{Z}}$ & $L_{\mathrm{X}} \cdot L_{\mathrm{Y}}$ & $L_{\mathrm{X}} \cdot L_{\mathrm{Z}}$ & $L_{\mathrm{X}} \cdot L_{\mathrm{Y}}$ & $L_{\mathrm{X}} \cdot L_{\mathrm{Y}}$ \\
\hline PGN-Sym & 28.50 & 29.61 & 24.06 & 29.61 & 24.06 & 24.06 \\
\hline PGN-Asym & 29.53 & 30.63 & 23.65 & 30.63 & 23.65 & 23.65 \\
\hline \multicolumn{7}{|c|}{ equivalent area $\left(\mathrm{nm}^{2}\right)$} \\
\hline & $2 * L_{\mathrm{Y} . \mathrm{t}}$ & $2^{*} L_{\mathrm{X} . \mathrm{t}}$ & $2 \pi \mathrm{Dt}$ & $2 * L_{X} . \mathrm{t}$ & $2 \pi \mathrm{Dt}$ & $2 \pi \mathrm{Dt}$ \\
\hline PGN-Sym & 3.27 & 3.40 & 1.78 & 3.40 & 1.78 & 1.78 \\
\hline PGN-Asym & 3.25 & 3.37 & 1.78 & 3.37 & 1.78 & 1.78 \\
\hline
\end{tabular}


(a)

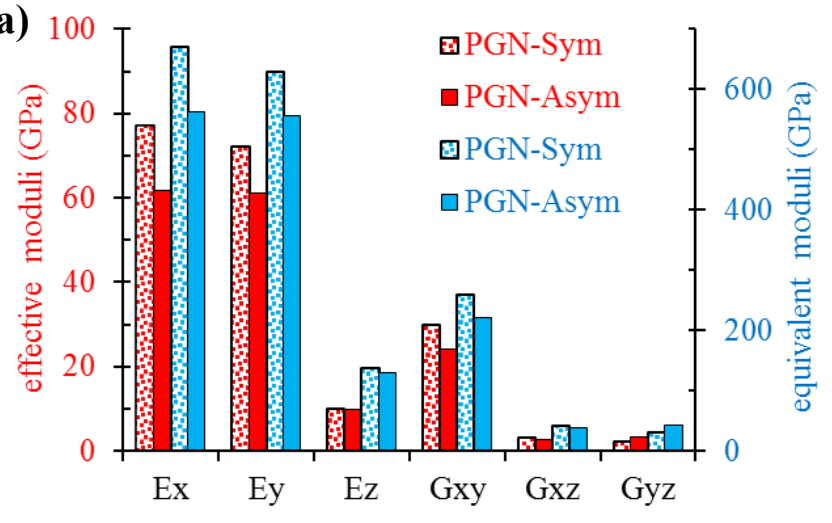

(b)

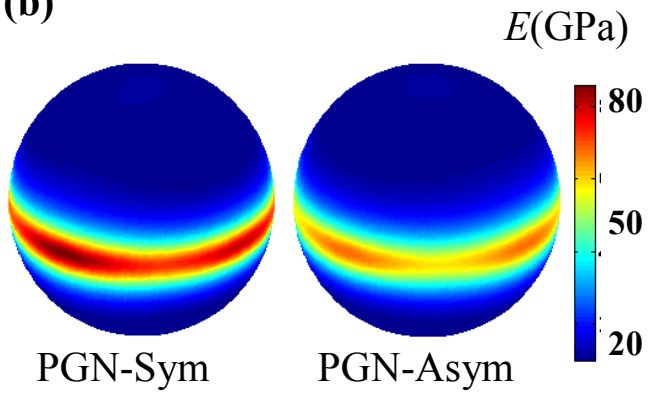

(c) effective area

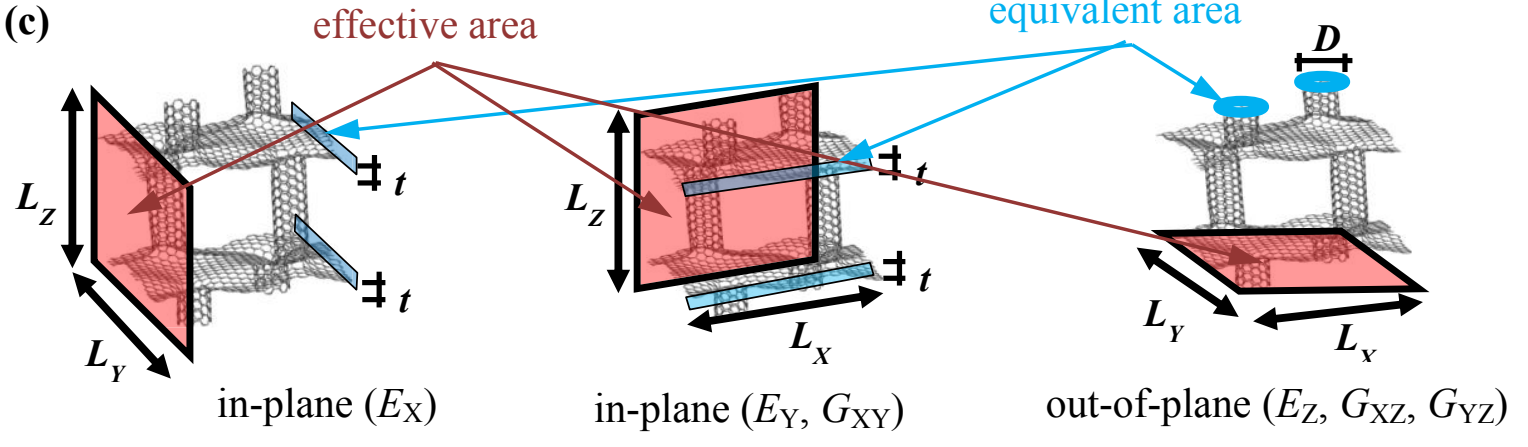

Figure 3. (a) Effective and equivalent moduli of PGN-Sym and PGN-Asym. Equivalent values are calculated based on atomic area, neglecting the voids. (b) Variation of the Young's modulus is shown for both PGN with a unit radius sphere. Each point on a sphere represents a vector passing through the center of the sphere with the color showing the value of the Young's modulus. (c) The effective and equivalent areas for each direction are shown. In the planar (outof-plane) directions, only the sheets (tubes) are resistive to the force in equivalent area.

A similar observations and trends to elasticity of PGN with effective areas are also valid for equivalent moduli. In this case, the thickness of CNT and the single graphene sheet are assumed to be $0.34 \mathrm{~nm}[39]$. As a consequence, the elastic moduli are drastically increased. The in-plane equivalent Young's moduli of PGN prototypes $\sim 0.5-0.7 \mathrm{TPa}$ are comparable to that of 
single graphene sheet $(>1 \mathrm{TPa})$ [40], the lower values stem from the wavy sheets and relatively flexible junctions, which introduce compliance in the system, thus decreasing the elastic moduli. However, the out-of-plane Young's moduli $\sim 0.13 \mathrm{TPa}$ are still $\sim 10$ times smaller than that of single CNT [41]. This low stiffness comes from the low out-of-plane flexibility of the graphene sheets [42], which contribute to the out-of-plane moduli of PGN due to discontinuity of the nanotubes. Simply put, in the out of plane direction, the tube and sheet can be considered as two springs that are serially connected. Thus, it is the lower stiffness (i.e. graphene) that will be first activated upon loading in the out of plane direction, hence a lower overall effective stiffness. Note that since elasticity is mainly about perturbation around the equilibrium state, tubes will not contribute much to the effective stiffness. Instead, their contribution will be manifest beyond elasticity regime, which will be discussed later.

The in-plane and out-of-plane Poisson's ratios of PGN-Sym and PGN-Asym are calculated from stiffness matrices (Eqs. 7-8) [43]. While both PGNs exhibit somewhat similar out-of-plane Poisson's ratios (Table 3), PGN-Asym's in-plane Poisson's ratio $\left(v_{\mathrm{xy}}\right)$ is significantly lower (zero or even slightly negative). Note that our calculated positive Poisson's ratio for PGN-Sym is in contrast to the negative Poisson's ratio previously calculated for a symmetric PGN using finite elements simulations [12]. We believe that this discrepancy is due to the inaccuracy of finite elements method and the associated empirical parameterizations of the elements in predicting material properties at the molecular level where MD is naturally a more reliable choice. Furthermore, we ascribe the zero (and perhaps negative) in-plane Poisson's ratio of PGN-Asym to its wrinkled sheets, similar to what has been observed in Pillared Boron Nitride nanostructure with asymmetric junctions and wrinkled sheets [43]. More precisely, upon loading along a certain direction, the wrinkled sheets flatten out in all the directions, thus bringing about 
a tensile strain in competition with the compressive strain transverse to the applied tensile loading (movies S1). Consequently, PGN-Asym might be better choice than PGN-Sym for fabrication of nanomaterials that require zero in-plane Poisson's ratio or auxetic nanomaterials which expand (shrink) laterally upon pulling (pushing) [44], with potential applications in sieving, strain sensing, gas separation and shock energy absorption [44, 45].

Table 3 - In-plane and out-of-plane Poisson's ratios of PGNs.

\begin{tabular}{llll} 
& $v_{x y}$ & $v_{x z}$ & $v_{y z}$ \\
\hline PGN-Sym & 0.106 & 0.095 & 0.101 \\
PGN-Asym & -0.020 & 0.111 & 0.122
\end{tabular}

\subsection{Inelastic Deformation Mechanism, Strength and Toughness}

Mechanical responses beyond elasticity can provide essential information on deformation mechanisms, which control strength and toughness preceding the materials failure. We studied the stress-strain behavior of PGNs until failure using MD simulations. Equivalent areas are used to calculate the stresses to better understand the underlying physics and compare the results with pure parent structures. Figure 4 show the stress-strain results for the two PGN prototyps along with average $\mathrm{C}-\mathrm{C}$ bond strains and stress contour plots. To our knowledge, this work is the first report on full stress-strain behaviors of any PGN structure until failure. For comparison, Figure 4a-c also shows the stress-strain plots of pure CNT and graphene, obtained from identical method and force field potential. First, we will discuss interesting (common) deformation mechanisms in both PGN-Sym and PGN-Asym and then highlight their differences. 
3.4.1 Out-of-Plane Deformation: a Two-Regime Synergistic Deformation. In view of the data in Figure $4 \mathrm{a}$, the ultimate strain of both PGNs $\left(\varepsilon_{U} \approx 42 \%\right)$ along the out-of-plane direction is $\approx 14 \%$ larger than that of pure $\mathrm{CNT}\left(\varepsilon_{U} \approx 28 \%\right)$ while maintaining similar ultimate stresses, $\sigma_{U} \approx 110 \mathrm{GPa}$. This significant stretching capacity stems from cooperation of two distinct deformation regimes (see movie S3). The first regime consists of mainly the out-of-plane deformation of the sheets, i.e. the geometrical re-arrangements of the graphene rings in the vicinity of the junctions. In this regime, although tubes are slightly strained, the geometric (rigid body type) displacement of the in-plane carbon rings (sheets) contributes most to the out-ofplane deformation while bearing small actual $\mathrm{C}-\mathrm{C}$ bond strains. However, C-C bonds in the octagonal rings (junctions) are highly strained to pass the imposed vertical strain to the horizontal sheets. This can be understood from the averaged bond strains in Figure $4 \mathrm{~b}-\mathrm{c}$ and stress concentrations (red dots) in Figure 4d-e. In the case of PGN-Sym (PGN-Asym), the heptagonal (octagonal) ring capacities become exhausted at $\varepsilon \approx 31 \%(\varepsilon \approx 26 \%)$ and their bond strains remain almost unchanged. At this point, which is the onset of the second regime (backbone stretching), the majority of the strains start to be transferred to the CNT. In this second regime, the actual $\mathrm{C}-\mathrm{C}$ bonds in the CNT tubes are significantly stretched until final failure at $\varepsilon_{U} \approx 42 \%$. As indicated in Figure $4 \mathrm{~b}-\mathrm{c}$ and movie $\mathrm{S} 3$ in SI, the non-hexagonal rings of the junctions always bear the maximum average strains, thus the junctions will act as the weakest link in the overall fracture. Figure 4d-e show highly stressed atoms, which are primarily at the junctions, causing the tubes to rip out of the graphene layers.

Together, the first and second deformation regimes in PGNs provide an out-of-plane toughness (area under the stress-strain curve) of $\approx 23.1 \mathrm{GPa}$ for PGN, which despite the lower stiffness, is comparable to that of pure CNT (23.0 GPa) (Figure 4a). Note that here "toughness" 
is defined as the amount of energy per volume a material absorbs before failure (representing the work-of-fracture) [46-48]. This definition is different from the classical "fracture toughness" with the unit of $\mathrm{Pa} \sqrt{m}$. The work-of-fracture is the area under the stress-strain curve and is profoundly affected by gradual, graceful fracture, whereas the "fracture toughness" does not incorporate this entire process [48]. We emphasize that it is the first deformation regime that provides the extra ductility and toughness. Interestingly, such a high toughness is not sacrificed by a low strength, which is typically expected in engineered materials [49-51]. This improved balance of strength and toughness along the out-of-plane direction of both PGNs is enabled by the cooperative behavior of tubes, junctions and out-of-plane flexibility of the sheets, and is a significant result of this paper. A somewhat similar cooperative effect in improving mechanical properties are reported for nanocarbons of different dimensionalities [52].

3.4.2. In-Plane Deformations. While PGN prototypes and graphene exhibit a somewhat similar in-plane stress-stress behavior (Figure 4f,k), it appears that the localized nature of strains on the junctions of PGNs alters the in-plane failure mechanism compared to that of graphene. More precisely, by strain localization around the octagonal or heptagonal rings, the cracks in PGNs initiate from these points and propagate at an inclined angle with respect to the direction of applied load (see dashed lines in Figure 4i,j and Figure 4n,o).

Within the in-plane directions of PGN, the ultimate stress, $\sigma_{U} \approx 120 \mathrm{GPa}$, in the zigzag (Y) direction is larger than $\sigma_{U} \approx 100 \mathrm{GPa}$ in the $\operatorname{armchair}(\mathrm{X})$ direction (c.f. Figure 4f,k). This disparity, which in part stems from the bond (zigzag versus armchair) orientations, is also observed in our MD simulation of graphene, and is verified by DFT calculations on graphene [53]. However, there is another mechanism that contributes to this difference in PGN prototypes: 
the heptagonal and octagonal rings, which are the hot spots under mechanical loading, undergo different stresses in armchair versus zigzag directions. For example, when the PGN-Asym is under axial strain in the zigzag direction, one third of all the octagonal rings (symbolized by $S_{\min }$ in Figure 4j) are furthest away from the stress concentration points and thus bear minimum stresses (Figure 5a). However, when the PGN-Asym is strained along the armchair direction, these same octagonal rings (symbolized by $S_{\max }$ in Figure 4o) are exactly coincided with the stress concentration points and thus have to sustain maximum stresses (the rest of the octagons bear somewhat similar stresses regardless of the direction of applied strain). Therefore, both bond orientation and stress concentrations in PGN-Asym lead to smaller strength capacity along the armchair direction than zigzag direction. A similar observation is valid for PGN-Sym. 

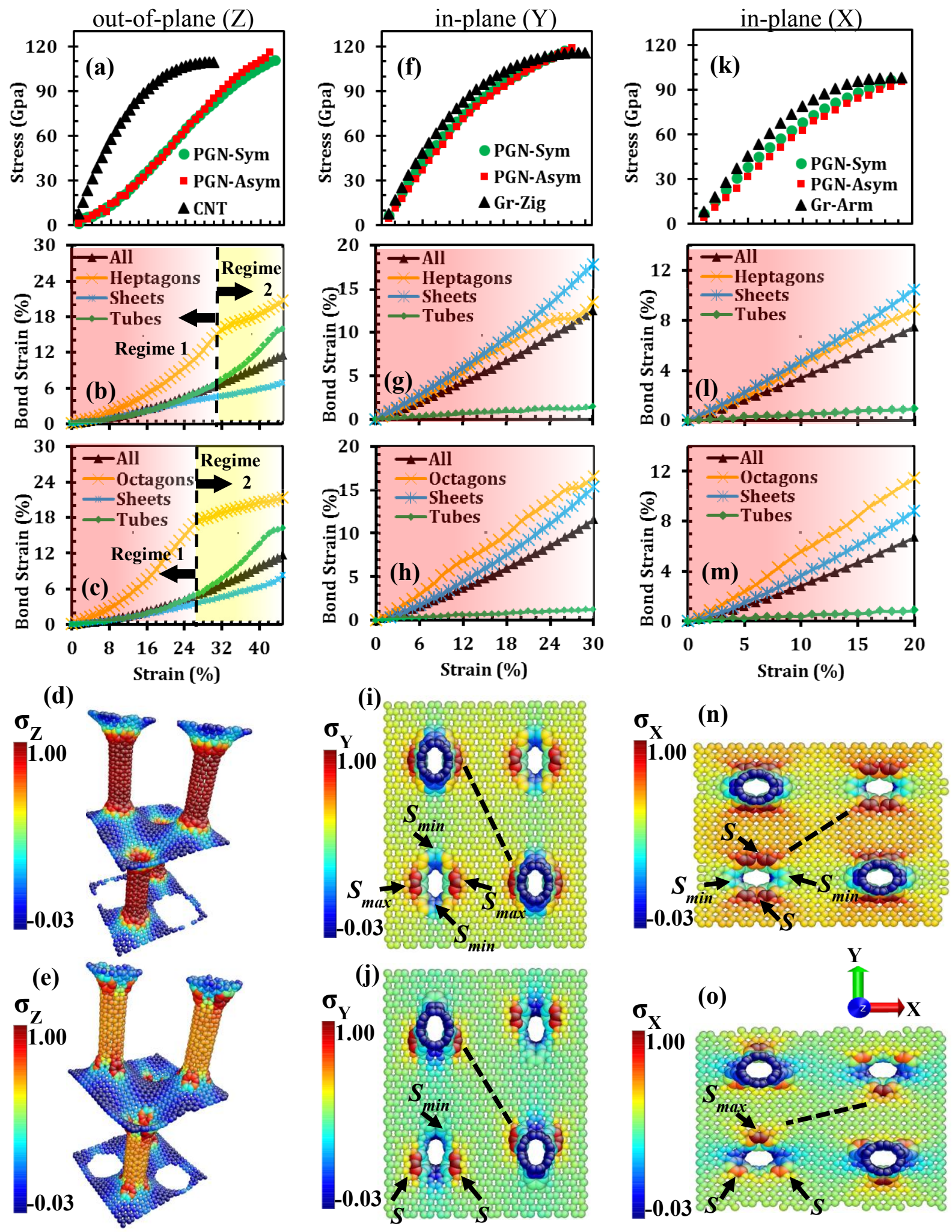
Figure 4. (a) Stress-strain plot of PGN-Sym and PGN-Asym along the Z-axis enabled by a tworegime deformation mechanism. (b-c) Average C-C bond strains of PGN-Sym (b) and PGNAsym (c) as a function of external strain, $\varepsilon_{Z} \cdot($ d-e) Stress contours for PGN-Sym (d) and PGNAsym (e) along the Z-axis at $\varepsilon_{Z} \approx 35 \%$ (Regime 2). (f-k) Stress-strain plots of PGNs and graphene along Y- (zigzag), and X- (armchair) axes. (g-l) Average C-C bond strains of PGNSym as a function of external strain. (h-m) Average C-C bond strains of PGN-Asym as a function of external strain. Interestingly, octagons are strained more than the sheets. (i-n) Stress contours along $\mathrm{Y}$ and $\mathrm{X}$ axes of PGN-Sym at $\varepsilon \approx 12 \%$. (j-o) Stress contours along $\mathrm{Y}$ and $\mathrm{X}$ axes of PGN-Asym at $\varepsilon \approx 12 \%$. The dashed lines in $(\mathrm{i}, \mathrm{j}, \mathrm{n}, \mathrm{o})$ denote the primary direction of crack propagations from the stress concentration points. The stresses in $(\mathrm{d}, \mathrm{e}, \mathrm{i}, \mathrm{j}, \mathrm{n}, \mathrm{o})$ are normalized to the highest stress at the ultimate strain in each direction.

3.4.3 Effect of Junction Type on Deformation Mechanisms. Our results in Figure 4 denote that while the symmetric vs asymmetric junction does not significantly alter the ultimate strain, strength, and toughness of PGN prototypes, their deformation and failure mechanisms are different in the two structures. In the out-of-plane direction, the first deformation regime of PGN-Asym ends at $\varepsilon \approx 26 \%$, which is $\sim 16 \%$ lower strain than that of PGN-Sym. This indicates that the three octagons in PGN-Asym have lower out-of-plane straining capacity than six heptagons in PGN-Sym (c.f. Figure 4b-c). This lower capacity causes PGN-Asym to fail at slightly $(\sim 2 \%)$ smaller strain than PGN-Sym (Figure $4 a)$. In planar directions, there is a more interesting difference between the two PGNs, i.e. the octagons are strained more than the sheets in PGN-Asym, in contrary to the trend in PGN-Sym (c.f. Figure 4g\&h or Figure 41\&m). Therefore, unlike the out-of-plane direction, octagonal rings in the asymmetric junctions bear 
more strains and provide higher strain capacity than heptagonal rings in the symmetric junctions. This extra $\mathrm{C}-\mathrm{C}$ bond straining in octagon rings results in slightly $(\sim 2 \%)$ higher ultimate strain in PGN-Asym in the planar directions than PGN-Sym. This behavior can be better explained via the notion of stress/strain concentrations. Figure 5 shows a schematic picture of a graphene sheet with an idealized circular hole under tensile load in $\mathrm{X}$ and $\mathrm{Y}$ directions. The triangular-shape junction and three octagons of PGN-Asym are also schematically superimposed in Figure 5a-b. In each $\mathrm{X}$ and $\mathrm{Y}$ direction, the locations of minimum and maximum stress points are marked by $C_{\max }$ and $C_{\min }$ using basic mechanics of materials [54]. From this figure, it is observed that when the tensile load is along the Y-direction (Zigzag), one out of three octagons coincides with the best possible position to bear the minimum stress $\left(C_{\min }\right)$. However, when the applied load is in the $\mathrm{X}$ (armchair) direction, the same octagon is exactly coincided with the stress concentration points $\left(C_{\max }\right)$ to bear the maximum stress.

In the case of PGN-Sym (Figure 5c-d), analogous superposition and conclusions are valid. For instance, while along the zigzag (Y) direction, two out of the six heptagons are maximally stressed, four out of the six heptagons are nearly (not exactly) maximally stressed along the armchair direction. Note that in the case of PGN-Sym, since the number of heptagons (6) is larger than octagons (3) in PGN-Asym, the stress/strain concentrations are more equally divided, resulting in lower strain values in heptagons. This explains why octagons in PGN-Asym bear more strains in the planar directions than heptagons in PGN-Sym, surpassing the average CC bond strain of the sheets in PGN-Asym. 


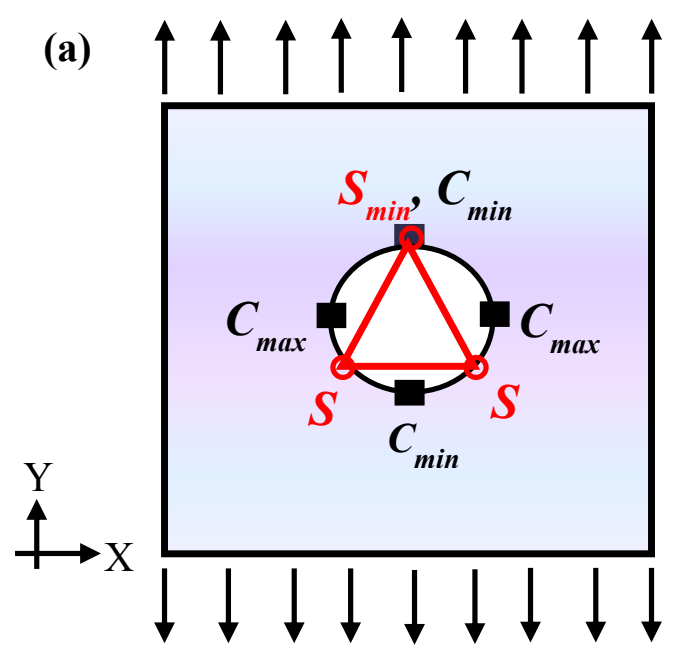

(b)
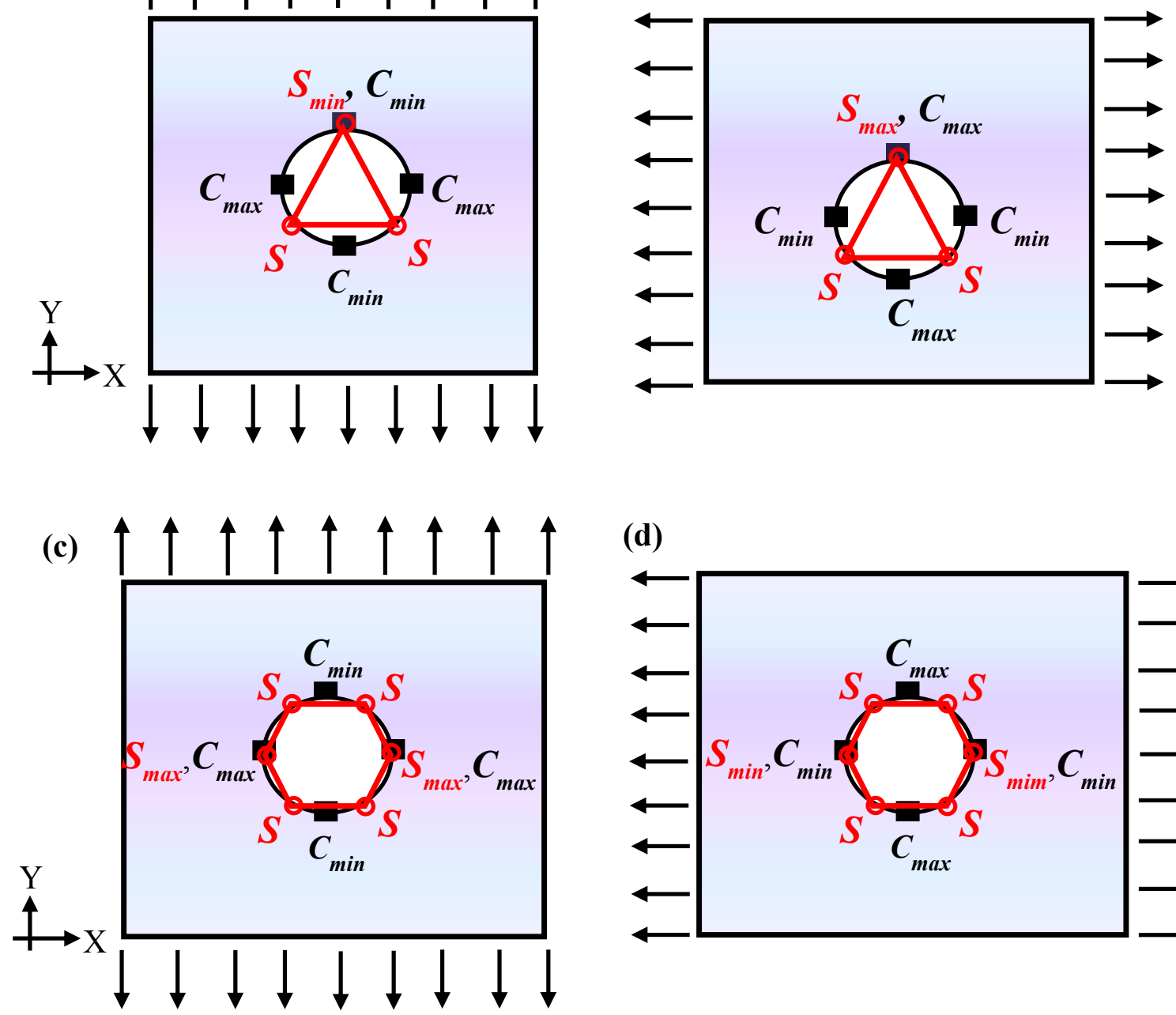

(d)

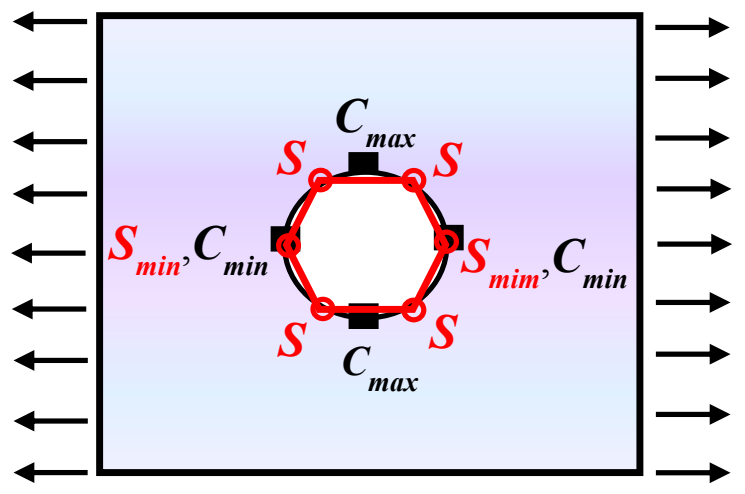

Figure 5. (a) and (b) Schematic picture of stress concentration points in a PGN-Asym junction under a tension load in $\mathrm{Y}$ and $\mathrm{X}$ direction. The black squares represent the locations of maximum and minimum stresses in an idealized circular hole under tensile loads. The small red circles denote the location of six octagons of PGN-Asym, which are superimposed to the circular hole. (c) and (d) Schematic picture of stress concentration points in a PGN-Sym junction under a tension load in $\mathrm{Y}$ and $\mathrm{X}$ direction.

\subsection{Discussions}


Given the wealth of existing literature on hybrid carbon based materials and the importance of these materials on pioneering technological advances, in this section we will discuss the key novelties and insights of this work along with necessary future studies from a materials design perspective. The discussions are summarized in four key parts as follows

- While Section 1 (Introduction) already has referred to and discussed the key reports on mechanical properties of $3 \mathrm{D}$ carbon materials, to our knowledge there has been no rigorous and complete study such as the present work on the effect of symmetric versus asymmetric junction topologies on the elastic and inelastic properties of PGN including full elastic tensors, bond strains, strength, ductility and toughness in all three-dimensional directions. Each work in the literature has typically addressed a limited feature or functionality of PGN along a certain direction with restricted implications.

- Identification of a two-regime deformation mechanism is a key novel aspect of this work that has been overlooked in previous studies. Understanding and tuning this cooperative deformation mechanism provides new insights on how to fuse various columns and sheets via covalent junctions to provide larger ductility and toughness without scarifying the strength. This is an interesting attribute that is highly desired in synthetic materials because toughness and strength are antagonistic mechanical properties in synthetic materials. Our work quantifies and clearly demonstrates how the synergies of CNTs, junction types and graphene sheets provide new degrees of freedom to address one of the key fundamental challenges of synthetic materials: that is the conflict between strength and toughness. This by itself is a key finding that provides new insights on how to strategize and design de novo 3D carbon-based nanomaterials toward this long engineering pursuit in materials. 
- Our work for the first time demonstrates that depending on the topology of the defect in the graphene sheets, the junction of PGN may end up being symmetric or asymmetric, which cause different elastic properties and fracture mechanisms. For instance, as shown in Figure 2a, asymmetric junctions lead to wrinkled (wavy) graphene sheets, providing $\sim 15-20 \%$ higher compliance (lower stiffness) along the in-plane directions as compared to their counterparts in straight graphene sheets with symmetric junctions (compare Ex, and Ey for PGN-Sym and PGNAsym in table 2). Similarly, the topology of symmetric versus asymmetric junctions influences the fracture processes that can be important from a material design perspective. For instance, as shown in Figure $4 \mathrm{~b}-\mathrm{c}$, the asymmetric junctions lower the critical strain between deformation regime 1 and 2 by $\sim 16 \%$, causing the tubes to be stressed more quickly than in the case of symmetric junctions (compare the start of regime 2 in Figure $4 b-c$ ). From a design perspective and considering that real CNTs have defects, the asymmetric junctions may not allow to utilize the full ductility and toughness capacity of PGN since the backbone of the tubes will be rapidly exposed to the external strain (regime 2). Therefore, when it comes to designing pillared graphene, it is important to ensure that the defected graphene sheet and the CNT type (armchair or zigzag) end up in a symmetric junction. However, if fracture and ductility is not an issue and more compliance and zero (negative) Poisson's ratios are desired, then asymmetric junctions are the preferred choice.

- We deliberately performed $\mathrm{MD}$ to the idealized limit of behavior at $\mathrm{T}=0 \mathrm{~K}$ via quasi-static loading to exclude thermally activated processes and strain rate effects. This might introduce some inconsistencies for comparison with experiments, materials design and functionality, which are typically subject to room temperature and finite strains. However, in view of the existing literature on the effects of temperature and strain rate on carbon based nanomaterials [55-58], we expect that mechanical properties, in particular fracture strength and fracture strain, will be reduced by increasing temperature and/or decreasing strain rate. In the case of temperature, this 
behavior is primarily because of the increased kinetic energy, more bond displacements, and defect formation associated with the higher temperatures. In the case of strain, the bond displacements/reconfigurations do have ample times to re-arrange themselves to lower energy states in slow strain rates, thus preventing the system to be on high-energy or metal stable local states, which cause unrealistically high mechanical properties. Furthermore, in the context of PGN, one may expect that there will be competition between the defects and grain boundaries in $\mathrm{CNT}$ and graphene versus those of the (a)symmetric junctions and potential impurities (Fe, Ni, etc.) of the junctions in controlling the mechanical properties. A detailed and unified understanding of the effect of temperature and strain rates on PGN requires a separate study, which is beyond the focus of the present manuscript.

Broadly, the above insights, novelties and discussion can be applied to other low-dimensional materials, such as Boron Nitride nanotubes/sheets, Molybdenum/Tunsgten disulphide (MoS2/WS2) or the combination thereof, towards creating multifunctional 3D nanomaterials with mutable mechano-electrothermo-optical properties [59].

\section{Conclusions}

We probed the effect of symmetric versus asymmetric junctions in controlling deformation mechanisms, and elastic and inelastic properties of pillared graphene via firstprinciples and molecular dynamics simulations. Unlike the stacked of graphene sheets, which exhibit weak out-of-plane properties, both junction types result in substantial improvements in the out-of-plane mechanical properties of PGN via a two-regime deformation mechanism. In this context, a synergistic geometrical feature is manifest via cooperation of junctions, tubes and sheets in the $3 \mathrm{D}$ architecture. This unique feature can overcome the intrinsic limitations of the 
1D and 2D constituents of PGN and impart superior properties including 3D balance of strength and toughness as well as an outstanding $\sim 42 \%$ out-of-plane ductility preceding the failure. We demonstrated that asymmetric junctions results in wrinkled sheets, which cause extra in-plane flexibility and shear compliance as well as a nearly zero/negative in-plane Poisson's ratio in PGN, thereby providing a new degree of freedom to control elastic properties. Although the symmetric and asymmetric junctions do not significantly alter the ultimate strain, strength, and toughness of PGN prototypes, the failure mechanisms are different in the two structures. The octagonal rings in PGN with asymmetric junctions bear more strains than the sheets, and act as hotspots for initiation of the fracture; a behavior that may not be present in PGN with symmetric junctions, owing to its symmetrically distributed strain concentrations in the heptagonal rings. To our knowledge, this work is the first report on the full stress-strain behavior of any PGN structure.

Broadly, our findings lay the foundation for discovering and studying numerous junction-induced hybrid 3D carbon architectures by fusing low dimensional building blocks including 0D fullerene, 1D nanotubes and 2D nanosheets. A rich set of hybrid carbon nanostructures have been developed and studied over the past years. However, similar advances for studying the effects of junctions have thus far remained elusive. To the best of our knowledge, this paper for the first time provides an atomistic "lens" to explore the diverse junction-mediated mechanical properties of PGN. This study potentially opens up a new phase space to tune hybrid carbon-based nanoelectronics and also can impact knowledge-based use of such nanomaterials as 3D fillers to modulate hybrid functional composites and layered materials [60-63]. 


\section{Supporting Information:}

A movie of in-plane deformation of PGN-Asym along with stress contours $\left(\sigma_{\mathbf{X}}\right)$. A movie of in-plane deformation of PGN-Sym along with stress contours $\left(\boldsymbol{\sigma}_{\mathbf{X}}\right)$. A movie of out-ofplane deformation of PGN-Asym along with stress contours $\left(\boldsymbol{\sigma}_{\mathbf{Z}}\right)$. In all movies, the blue and red colors denote respectively low and high atomic stresses along the pulling direction obtained from virial theorem.

\section{Acknowledgments}

This work was supported in part by Rice University and in part by NSF grant number CMMI-1235522. The supercomputer machines utilized in this work were supported in part by NIH award NCRR S10RR02950 and an IBM Shared University Research (SUR) Award in partnership with CISCO, Qlogic and Adaptive Computing, and in part by the Data Analysis and Visualization Cyber infrastructure funded by NSF under grant OCI-0959097.

\section{Author Contributions}

R.S. designed the research; N.S and R.S performed the computational and experimental research; N.S. and R.S analyzed the data; and R.S. wrote the paper.

\section{Competing Financial Interest}

The authors declare no competing financial interest.

\section{REFERENCES}

[1] De Volder MFL, Tawfick SH, Baughman RH, Hart AJ. Carbon Nanotubes: Present and Future Commercial Applications. Science. 2013;339(6119):535-9. 
[2] Lee C, Wei X, Kysar JW, Hone J. Measurement of the Elastic Properties and Intrinsic Strength of Monolayer Graphene. Science. 2008;321(5887):385-8.

[3] Huang X, Yin Z, Wu S, Qi X, He Q, Zhang Q, et al. Graphene-Based Materials: Synthesis, Characterization, Properties, and Applications. Small. 2011;7(14):1876-902.

[4] Thostenson ET, Ren Z, Chou TW. Advances in the science and technology of carbon nanotubes and their composites: a review. Composites Science and Technology. 2001;61(13):1899-912.

[5] Hornbaker DJ, Kahng S-J, Misra S, Smith BW, Johnson AT, Mele EJ, et al. Mapping the One-Dimensional Electronic States of Nanotube Peapod Structures. Science. 2002;295(5556):828-31.

[6] Lee J, Kim H, Kahng SJ, Kim G, Son YW, Ihm J, et al. Bandgap modulation of carbon nanotubes by encapsulated metallofullerenes. Nature. 2002;415(6875):1005-8.

[7] Okada S, Saito S, Oshiyama A. Energetics and Electronic Structures of Encapsulated C_\{60\} in a Carbon Nanotube. Physical Review Letters. 2001;86(17):3835-8.

[8] Nasibulin AG, Pikhitsa PV, Jiang H, Brown DP, Krasheninnikov AV, Anisimov AS, et al. A novel hybrid carbon material. Nat Nano. 2007;2(3):156-61.

[9] Wu X, Zeng XC. Periodic graphene nanobuds. Nano Lett. 2009;9(1):250-6.

[10] Ding F, Lin Y, Krasnov PO, Yakobson BI. Nanotube-derived carbon foam for hydrogen sorption. The Journal of Chemical Physics. 2007;127(16):164703-6.

[11] Dimitrakakis GK, Tylianakis E, Froudakis GE. Pillared Graphene: A New 3-D Network Nanostructure for Enhanced Hydrogen Storage. Nano Letters. 2008;8(10):3166-70.

[12] Sihn S, Varshney V, Roy AK, Farmer BL. Prediction of 3D elastic moduli and Poisson's ratios of pillared graphene nanostructures. Carbon. 2012;50(2):603-11. 
[13] Varshney V, Patnaik SS, Roy AK, Froudakis G, Farmer BL. Modeling of Thermal Transport in Pillared-Graphene Architectures. ACS Nano. 2010;4(2):1153-61.

[14] Wang C-H, Fang T-H, Sun W-L. Mechanical properties of pillared-graphene nanostructures using molecular dynamics simulations. Journal of Physics D: Applied Physics. 2014;47(40):405302.

[15] Niu J, Li M, Xia Z. Growth mechanisms and mechanical properties of 3D carbon nanotube-graphene junctions: molecular dynamic simulations. RSC Advances. 2014;4(64):33848-54.

[16] Sasaki H, Hagi T, Shintani K. Mechanical Properties of Pillared-Graphene Nanostructures under Shear Loads. Materials Research Society Proceeding. Cambridge Cmbridge University Press; p. mrsf12-1505-w10-27.

[17] Wesolowski RP, Terzyk AP. Pillared graphene as a gas separation membrane. Physical Chemistry Chemical Physics. 2011;13(38):17027-9.

[18] Novaes FD, Rurali R, Ordejón P. Electronic Transport between Graphene Layers Covalently Connected by Carbon Nanotubes. ACS Nano. 2010;4(12):7596-602.

[19] Paul RK, Ghazinejad M, Penchev M, Lin J, Ozkan M, Ozkan CS. Synthesis of a Pillared Graphene Nanostructure: A Counterpart of Three-Dimensional Carbon Architectures. Small. 2010;6(20):2309-13.

[20] Lin J, Zhong J, Bao D, Reiber-Kyle J, Wang W. Supercapacitors Based on Pillared Graphene Nanostructures. Journal of Nanoscience and Nanotechnology. 2012;12(3):1770-5.

[21] Park J, Prakash V. Phonon scattering and thermal conductivity of pillared graphene structures with carbon nanotube-graphene intramolecular junctions. Journal of Applied Physics. 2014;116(1):014303. 
[22] Chih-Hao W, Te-Hua F, Wei-Li S. Mechanical properties of pillared-graphene nanostructures using molecular dynamics simulations. Journal of Physics D: Applied Physics. 2014;47(40):405302.

[23] Loh GC, Teo EHT, Tay BK. Tuning the Kapitza resistance in pillared-graphene nanostructures. Journal of Applied Physics. 2012;111(1):013515-6.

[24] Baowan D, Cox BJ, Hill JM. Joining a carbon nanotube and a graphene sheet. 2008:5-8.

[25] Parr RG, Yang W. Density-Functional Theory of Atoms and Molecules: Oxford University Press, USA 1994.

[26] Plimpton S. Fast Parallel Algorithms for Short-Range Molecular Dynamics. J Comput Phys. 1995;117(1):1-19.

[27] Stuart SJ, Tutein AB, Harrison JA. A reactive potential for hydrocarbons with intermolecular interactions. The Journal of Chemical Physics. 2000;112(14):6472-86.

[28] Grantab R, Shenoy VB, Ruoff RS. Anomalous Strength Characteristics of Tilt Grain Boundaries in Graphene. Science. 2010;330(6006):946-8.

[29] Wei Y, Wu J, Yin H, Shi X, Yang R, Dresselhaus M. The nature of strength enhancement and weakening by pentagon-heptagon defects in graphene. Nat Mater. 2012;11(9):759-63.

[30] Nose S. A Unified Formulation of the Constant Temperature Molecular Dynamics Methods. J Chem Phys. 1984;81(1):511-9.

[31] Hoover WG. Canonical Dynamics: Equilibrium Phase-Space Distributions. Phys Rev A. 1985;31(3):1695-7.

[32] Humphrey W, Dalke A, Schulten K. VMD: Visual Molecular Dynamics. J Mol Graphics. 1996;14(1):33-8. 
[33] Li J. AtomEye: an Efficient Atomistic Configuration Viewer. Modell Simul Mater Sci Eng. 2003;11(2):173-7.

[34] Okada S, Saito S, Oshiyama A. Energetics and Electronic Structures of Encapsulated C60 in a Carbon Nanotube. Phys Rev Lett. 2001;86(17):3835-8.

[35] Shahsavari R, Buehler MJ, Pellenq RJM, Ulm F-J. First-Principles Study of Elastic Constants and Interlayer Interactions of Complex Hydrated Oxides: Case Study of Tobermorite and Jennite. J Am Ceram Soc. 2009;92(10):2323-30.

[36] Nye JF. Physical Properties of Crystals Oxford, England: Oxford University Press; 1957.

[37] Shahsavari R, Pellenq RJM, Ulm F-J. Empirical force fields for complex hydrated calciosilicate layered materials. PCCP. 2011;13(3):1002-11.

[38] Cromwell PR. Polyhedra: Cambridge University Press; 1997.

[39] Huang Y, Wu J, Hwang KC. Thickness of graphene and single-wall carbon nanotubes. Physical Review B. 2006;74(24):245413.

[40] Scarpa F, Adhikari S, Srikantha AP. Effective elastic mechanical properties of single layer graphene sheets. Nanotechnology. 2009;20(6).

[41] Krishnan A, Dujardin E, Ebbesen TW, Yianilos PN, Treacy MMJ. Young's modulus of single-walled nanotubes. Physical Review B. 1998;58(20):14013-9.

[42] Wang Q. Simulations of the bending rigidity of graphene. Physics Letters A. 2010;374(9):1180-3.

[43] Sakhavand N, Shahsavari R. Synergistic Behavior of Tubes, Junctions, and Sheets Imparts Mechano-Mutable Functionality in 3D Porous Boron Nitride Nanostructures. The Journal of Physical Chemistry C. 2014;118(39):22730-8. 
[44] Yang W, Li Z-M, Shi W, Xie B-H, Yang M-B. Review on Auxetic Materials. J Mater Sci. 2004;39(10):3269-79.

[45] Grima JN, Jackson R, Alderson A, Evans KE. Do Zeolites Have Negative Poisson's Ratios? Adv Mater. 2000;12(24):1912-8.

[46] Meyers MA, McKittrick J, Chen P-Y. Structural Biological Materials: Critical Mechanics-Materials Connections. Science. 2013;339(6121):773-9.

[47] Wegst UGK, Ashby MF. The Mechanical Efficiency of Natural Materials. Philos Mag. 2004;84(21):2167-86.

[48] Meyers MA, Chen P-Y, Lin AY-M, Seki Y. Biological Materials: Structure and Mechanical Properties. Prog Mater Sci. 2008;53(1):1-206.

[49] Ritchie RO. The Conflicts Between Strength and Toughness. Nat Mater. 2011;10(11):817-22.

[50] Sakhavand N, Muthuramalingam P, Shahsavari R. Toughness Governs the Rupture of the Interfacial H-Bond Assemblies at a Critical Length Scale in Hybrid Materials. Langmuir. 2013;29(25):8154-63.

[51] Sakhavand N, Shahsavari R. Universal composition-structure-property maps for natural and biomimetic platelet-matrix composites and stacked heterostructures. Nature communications. 2015;6:6523.

[52] Prasad KE, Das B, Maitra U, Ramamurty U, Rao CNR. Extraordinary Synergy in the Mechanical Properties of Polymer Matrix Composites Reinforced with 2 Nanocarbons. Proc Natl Acad Sci. 2009;106(32):13186-9. 
[53] Peng Q, Chen Z, De S. A Density Functional Theory Study of the Mechanical Properties of Graphane With van der Waals Corrections. Mechanics of Advanced Materials and Structures. 2014;22(9):717-21.

[54] Hearn EJ. Mechanics of Materials 2: The Mechanics of Elastic and Plastic Deformation of Solids and Structural Components. 2 ed: Butterworth-Heinemann Ltd; 1995.

[55] Daly M, Reeve M, Veer Singh C. Effects of topological point reconstructions on the fracture strength and deformation mechanisms of graphene. Computational Materials Science. 2015;97:172-80.

[56] Daly M, Veer Singh C. A kinematic study of energy barriers for crack formation in graphene tilt boundaries. Journal of Applied Physics. 2014;115(22):223513.

[57] Wei C, Cho K, Srivastava D. Tensile strength of carbon nanotubes under realistic temperature and strain rate. Physical Review B. 2003;67(11):115407.

[58] Zhao H, Aluru NR. Temperature and strain-rate dependent fracture strength of graphene. Journal of Applied Physics. 2010;108(6):064321.

[59] Sakhavand N, Shahsavari R. Dimensional Crossover of Thermal Transport in Hybrid Boron Nitride Nanostructures. ACS Applied Materials \& Interfaces. 2015.

[60] Rafiee MA, Narayanan TN, Hashim DP, Sakhavand N, Shahsavari R, Vajtai R, et al. Hexagonal Boron Nitride and Graphite Oxide Reinforced Multifunctional Porous Cement Composites. Adv Funct Mater. 2013;23(45):5624-30.

[61] Jalilvand S, Shahsavari R. Molecular Mechanistic Origin of Nanoscale Contact, Friction, and Scratch in Complex Particulate Systems. ACS Appl Mater Interfaces. 2015;7(5):3362-72. 
[62] Prakash M, Sakhavand N, Shahsavari R. H2, N2, and CH4 Gas Adsorption in Zeolitic Imidazolate Framework-95 and -100: $\mathrm{Ab}$ Initio Based Grand Canonical Monte Carlo Simulations. J Phys Chem C. 2013;117(46):24407-16.

[63] Shahsavari R, Chen L. Screw dislocations in complex, low symmetry oxides: core structures, energetics, and impact on crystal growth. ACS Appl Mater Interfaces. 2015;7(4):2223-34. 